\title{
CoviD-19 PPE-associated dermatoses: effect on work and wellbeing
}

\author{
Authors: Aarthy K Uthayakumar, ${ }^{\mathrm{A}}$ Evangelia Panagou, ${ }^{\mathrm{A}}$ Seshi Manam, ${ }^{\mathrm{B}}$ Anna Schauer, ${ }^{\mathrm{A}}$ Ophelia Veraitch, ${ }^{\mathrm{B}}$ \\ Steve Walker, ${ }^{B}$ Emma Edmonds, ${ }^{B}$ Jennifer Crawley ${ }^{B}$ and Claire Martyn-Simmons ${ }^{B}$
}

The COVID-19 pandemic has led to more stringent infection control practice within hospitals, with the added requirement of personal protective equipment (PPE). Consequentially, the incidence of PPE-related dermatoses in healthcare workers is increasing. Analysis of staff members seen in a dermatology self-referral service during the first COVID-19 peak revealed a wide range of healthcare professionals being affected, with irritant hand dermatitis and acneiform eruptions being the commonest diagnoses. These PPE-related dermatoses had significant effects on staff members' work and wellbeing, which improved following assessment and treatment by dermatology. Given the change to our behaviour wearing PPE daily, which is likely to continue for the foreseeable future, the consequences will inevitably affect both healthcare workers and the general public, with these common dermatoses presenting in both primary and secondary care. It is therefore imperative to recognise both the physical and emotional impact that COVID-19 PPE has, and initiate prompt treatment to improve wellbeing.

KEYWORDS: occupational dermatoses, wellbeing, PPE, hand dermatitis, dermatology

DOI: $10.7861 /$ fhj.2020-0210

\section{Introduction}

COVID-19 has led to several changes in hospital infection control practices, primarily requirement for wearing personal protective equipment (PPE). During COVID-19, up to $97 \%$ of healthcare workers experience skin reactions secondary to enhanced infection control measures.' Hand eczema is common in healthcare workers; risk factors include frequent handwashing and length of time wearing gloves. ${ }^{2,3}$ Furthermore, the negative impact of skin conditions on quality of life is increasingly appreciated. ${ }^{4}$

Authors: ${ }^{A}$ dermatology registrar, University College London Hospitals NHS Foundation Trust, London, UK; ${ }^{B}$ dermatology consultant, University College London Hospitals NHS Foundation Trust, London, UK

\section{Report}

A staff self-referral dermatology service commenced approximately 4 weeks into the first COVID-19 lockdown. The service offered a face-to-face dermatology consultation for any hospital staff experiencing skin conditions secondary to enhanced COVID-19 infection control. All staff members who had attended the dermatology clinic were emailed a voluntary anonymous online survey, which assessed the impact of their skin condition on their work and wellbeing.

A total of 67 staff members were seen over an 8-week period. The age range was $23-60$ years (median 34 years), $70 \%$ were aged 23-39 years. The female-to-male ratio was 4:1. Staff members were from several disciplines (Table 1), with nursing staff being most frequently represented (57\%).

The most prevalent dermatoses were irritant contact hand dermatitis (45\%), acne/folliculitis (24\%) and eczema flare-up (28\%) (Table 1). Thirteen patients had multiple diagnoses, the majority being a combination of irritant hand contact dermatitis and acne. One patient was diagnosed with Darier's disease, exacerbated by PPE. Of the eczematous dermatoses treated, $91 \%$ were of at least moderate severity requiring emollient and topical steroid treatment; $9 \%$ were mild and treated with emollients alone. Of the acneiform eruptions, 33\% required oral antibiotics and topical retinoids (Table 1).

There were $43(64 \%)$ responses to the questionnaire. $46.5 \%$ of respondents had a pre-existing skin condition, which in the majority did not have a significant adverse effect on their work or study $(80 \%)$ or wellbeing (60\%) prior to COVID-19.

With the use of PPE, there was a marked increase in the impact of skin conditions; $70 \%$ reported a significant adverse effect on their work or study, and $86 \%$ on wellbeing (Fig 1). Following dermatology consultation, $72 \%$ agreed that their skin had improved and $72 \%$ agreed that the degree to which their skin affected their wellbeing had reduced (Fig 2).

Analysis of free-text survey answers revealed three broad themes; physical symptoms, concerns over PPE, and emotions (predominantly anxiety, fear and self-consciousness).

Dryness and associated irritation, in addition to pain and pruritus, were commonly described symptoms and contributed to feelings of anxiety. Staff reported interrupted sleep and being 'unable to do much at home' due to skin irritation. As per infection control advice, alcohol gel was used frequently, which 


$\begin{array}{ll}\text { Table 1. Breakdown of } \mathbf{6 7} & \text { staff members assessed } \\ \text { in dermatology staff self-referral clinic by job role, } \\ \text { dermatosis and treatment }\end{array}$

'was painful'; however, switching to more frequent handwashing worsened their skin, making 'most tasks difficult to perform'.

Wearing PPE contributed negatively to work and wellbeing. One reported that 'wearing gloves became uncomfortable to the point I didn't want to wear them anymore'. Although taking frequent breaks is recommended, some reported that they felt the requirement of donning and doffing for each break meant 'a huge waste of PPE' and as such would stay in PPE for longer periods.

Staff reported feeling 'uncomfortable and self-conscious' and 'patients would comment', leading to feeling 'ashamed and embarrassed' with 'lowered self-esteem'. Some were 'fearful of being at work', and 'terrified of a second wave and being redeployed again which would worsen my skin'.

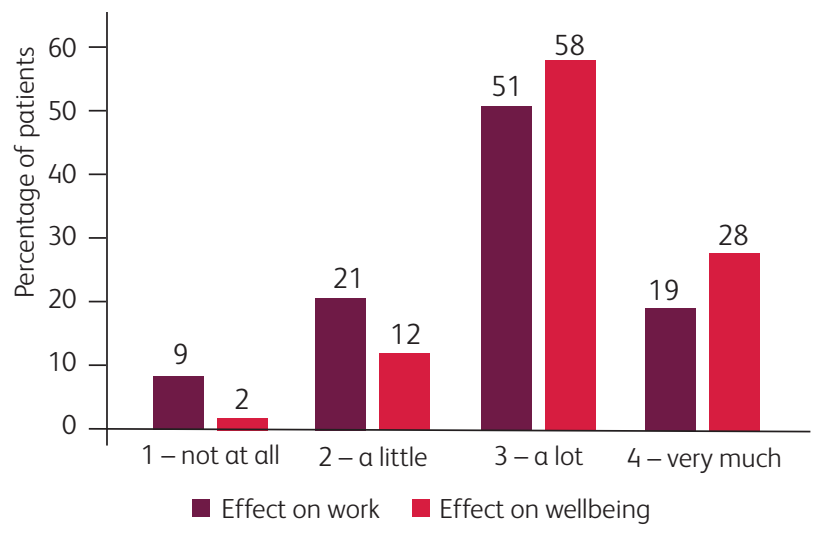

Fig 1. Patients' survey responses to the question 'During COVID-19, to what extent did your skin adversely affect your work and wellbeing?'

\section{Discussion}

The demographic data and prevalence of PPE-related dermatoses in healthcare workers in this study are similar to those reported in surveys performed in China. ${ }^{1.5}$ Lin et a ${ }^{5}$ reported that the commonest eruptions were dryness, papules or erythema and maceration, with the hands, cheeks and nasal bridge being the most commonly affected areas. ${ }^{5} \mathrm{~A}$ high rate of exacerbation of acne with $\mathrm{N} 95$ masks has also been reported. ${ }^{6}$ The commonest symptoms reported were dryness, pain and pruritus, ${ }^{1}$ as was found in our study. The occurrence of skin conditions corresponds to the duration of wearing PPE and working frequency. ${ }^{7}$ The majority of our self-referrals were nursing staff wearing PPE for long shifts.

The NHS England recommendation to change masks every 2 hours was often unknown to staff, who would frequently remain

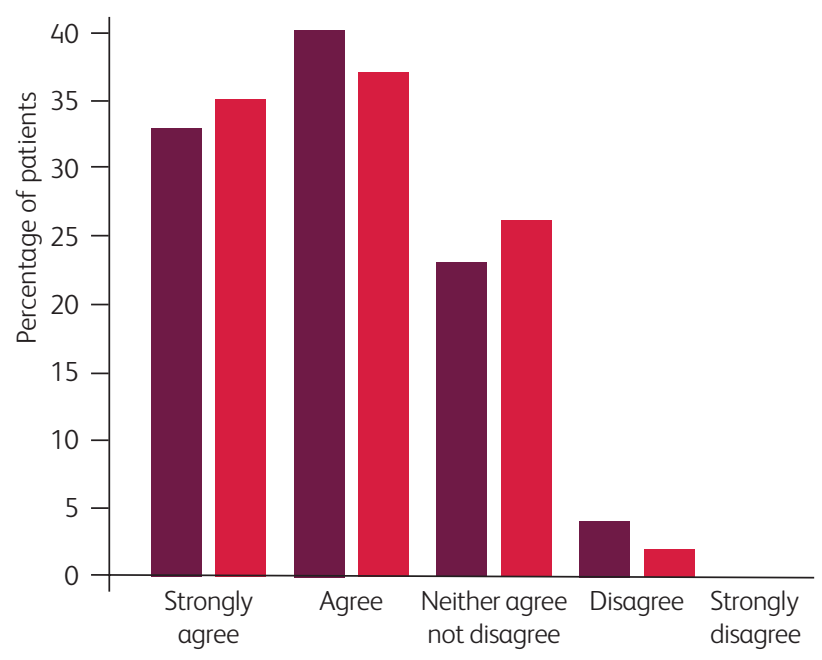

- The degree to which my skin has adversely affected my wellbeing has reduced

My skin has improved

Fig 2. Patients' survey responses to the question 'To what extent do you agree with these statements? Following dermatology consultation'. 
Ten tips for keeping skin healthy during COVID-19

$>$ Wash hands with soap followed by soap substitute and pat dry.

> Use a regular bland emollient to minimise hand dryness.

$>$ A greasy emollient under cotton gloves at night may be effective.

$>$ For red, itchy, inflamed areas of hand dermatitis, use topical corticosteroid of moderate potency if needed.

$>$ Change mask frequently, ideally every 2 hours.

$>$ Avoid greasy, comedogenic facial products (contribute to occlusion) to minimise acne.

> Face wash containing salicylic acid and/or benzoyl peroxide may be helpful with facial acne.

> For pressure injury from face masks, dressings (eg DuoDERM ${ }^{\circledR}$ Extra Thin) can be applied at the site of the seal.

> Applying emollient to the face 30 mins before donning PPE can help minimise pressure injury and facial dermatitis.

> $3 \mathrm{M}^{\mathrm{TM}}$ Cavilon $^{\mathrm{TM}}$ Barrier Cream application to pressure sites of FFP3 mask and face shield seals prior to application does not affect fit of seal and can help to protect the skin.

in level 2-3 PPE for several hours. In addition, concerns over PPE shortages may have led to staff remaining in PPE for longer than recommended and an exacerbation of occupational dermatoses.

Occupational contact dermatitis is known to have a significant impact on health-related quality of life and work loss. ${ }^{3}$ Studies have shown an increased risk of depression from hand eczema and acne. ${ }^{2,3,4}$ Furthermore, contact dermatitis affecting the hands can lead to a higher prevalence of bacterial colonisation, with further infection control implications. ${ }^{3}$ Our thematic analysis highlighted the negative impact of occupational dermatoses, with reported anxiety and lowered self-esteem. Although alcohol-based products have a high antimicrobial activity and lower risk of skin damage than handwashing, ${ }^{5}$ our study found that continued use of these caused extreme irritation and soreness.

Limitations in our study include response bias, as those with more severe PPE-related dermatoses, with a greater impact on work and wellbeing, may be more likely to respond. The Likert scale to assess the impact on work and wellbeing was based on the validated Dermatology Life Quality Index (DLQI) questionnaire; however, a full DLQI was not performed, and therefore specific aspects of wellbeing such as social activities and relationships were not evaluated. Anxiety and depression were not formally assessed in our survey.

\section{Conclusions}

Existing studies during COVID-19 have highlighted the high prevalence of skin damage in healthcare workers, which is corroborated in the findings from this study. Hand eczema is one of the commonest occupational diseases and, given the ongoing use of PPE and increased hand hygiene in both hospitals and public settings, we are likely to see an increase in these conditions, in both primary and secondary care. The negative impact of the COVID-19 pandemic on healthcare workers is multifactorial, with PPE-related dermatoses being one of many factors. Given the long-term ongoing use of PPE, attempts should be made to modify infection control practices and increase ease of access to treatment in order to minimise occupational injuries, which have both a physical and an emotional impact on healthcare workers.

\section{References}

1 Lan J, Song Z, Miao X et al. Skin damage among health care workers managing coronavirus disease-2019. J Am Acad Dermatol 2020;82:1215-6.

2 Cvetkovski RS, Zachariae R, Jensen H, Olsen J, Johansen JD, Agner T. Quality of life and depression in a population of occupationa hand eczema patients. Contact Dermatitis 2006;54:106-11.

3 Smedley J, Williams S, Peel P, Pedersen K. Management of occupational dermatitis in healthcare workers: a systematic review. Occup Environ Med 2012;69:276-9.

4 Dalgard FJ, Gieler U, Tomas-Aragones L et al. The psychological burden of skin diseases: a cross-sectional multicenter study among dermatological out-patients in 13 European countries. J Invest Dermatol 2015;135:984-91.

5 Lin P, Zhu S, Huang $Y$ et al. Adverse skin reactions among healthcare workers during the coronavirus disease 2019 outbreak: a survey in Wuhan and its surrounding regions. $\mathrm{Br}$ ] Dermatol 2020;183:190-2.

6 Zuo Y, Hua W, Luo Y, Li L. Skin reactions of N95 masks and medial masks among health-care personnel: a self-report questionnaire survey in China. Contact Dermatitis 2020;83:145-7.

7 Pei S, Xue Y, Zhao S et al. Occupational skin conditions on the front line: a survey among 484 Chinese healthcare professionals caring for Covid-19 patients. J Eur Acad Dermatol Venereol 2020;34:e354-7.

Address for correspondence: Dr Aarthy Kanmany Uthayakumar, University College London Hospital, 235 Euston Road, London NW1 2BU, UK.

Email: aarthy.uthayakumar@nhs.net 